\section{Cahiers d'ethnomusicologie}

Anciennement Cahiers de musiques traditionnelles

$10 \mid 1997$

Rythmes

\title{
Madagascar. Musique Antanosy
}

AIMP, 1997

Madeleine Leclair

\section{OpenEdition}

Journals

Édition électronique

URL : http://journals.openedition.org/ethnomusicologie/943

ISSN : 2235-7688

Éditeur

ADEM - Ateliers d'ethnomusicologie

Édition imprimée

Date de publication : 1 décembre 1997

Pagination : 358-361

ISBN : 2-8257-0579-9

ISSN : 1662-372X

Référence électronique

Madeleine Leclair, «Madagascar. Musique Antanosy », Cahiers d'ethnomusicologie [En ligne], 10 | 1997, mis en ligne le 06 janvier 2012, consulté le 21 avril 2019. URL : http://journals.openedition.org/ ethnomusicologie/943

Ce document a été généré automatiquement le 21 avril 2019

Tous droits réservés 


\title{
Madagascar. Musique Antanosy
}

AIMP, 1997

\author{
Madeleine Leclair
}

\section{RÉFÉRENCE}

Madagascar. Musique Antanosy. Enregistrements (août 1995) et textes de présentation de Victor Randrianary. 6 photos de Sylvie Rifflet et 1 carte. Livret de 23 pages français/ anglais. 1 CD AIMP IL, VDE-924, 1997.

1 Officiellement, on distingue aujourd'hui à Madagascar dix-huit groupes ethniques différents de par leur histoire et leur isolement ; tous cependant parlent la même langue, le malgache (pouvant présenter des variantes dialectales). Parmi les régions choisies par ces groupes, la partie sud-ouest de l'île a vu s'installer les populations Antandroy et Antanosy, pratiquant principalement la culture du riz et l'élevage de zébus. De ces deux populations, ce sont les Antanosy qui ont été les moins étudiés. Les rares publications discographiques faisant entendre des extraits de musique antanosy montrent pourtant que cette population est dépositaire d'une riche production musicale. Qu'il s'agisse de musique liée à des cultes de possession, à des rituels ou encore de musique profane, cette production musicale semble être principalement caractérisée par un instrumentarium et une utilisation de la voix donnant lieu à diverses formes de polyphonie.

Le disque Musique Antanosy réunit des morceaux choisis extraits des répertoires de cette population et en ce sens, il se situe dans la lignée des deux autres publications discographiques auxquelles Victor Randrianary a participé. Il est l'auteur du CD Anthologie des voix de Madagascar, publié en 1997, dans la collection Inédit - Maison des cultures du monde (W 260076), et a collaboré aux côtés d'Alain Desjacques à la réalisation du CD Madagascar. Pays Bara paru en 1996 chez Ocora (C 560089).

C'est dans une perspective de préservation du patrimoine culturel antanosy, dont l'authenticité, nous dit V. Randrianary, est fragilisée par le contact avec l'étranger, que l'auteur fixe les limites de sa publication. L'objectif sous-tendant la production de ce 
disque dans son ensemble est sommairement annoncé comme suit : il s'agit pour Victor Randrianary de «[...] constituer des archives de cette musique [Antanosy] qui puissent donner des références aux futures générations comme aux chercheurs ou aux simples amateurs de traditions musicales de Madagascar» (p. 4 du livret). Les extraits du disque sont à mon avis du plus grand intérêt musical, mais le contenu informatif du livret n'est pas toujours très utile quant à la compréhension des faits musicaux qu'il est censé éclairer. Le cheminement choisi par l'auteur pour nous présenter « l'immense diversité de la musique antanosy » (p. 4) ne ressort pas clairement de son texte d'introduction ni de la mise en page du livret, et l'auditeur a finalement du mal à entrevoir le lien entre les différents répertoires qui lui sont proposés.

4 Malgré cela, l'intérêt esthétique des exemples musicaux présentés est remarquable. Les quelques extraits des différents corpus de musique vocale sont du plus grand intérêt. La première plage du disque nous fait entendre un chant (alternance entre une soliste et un ensemble de voix mixtes) exécuté lors des cérémonies de circoncision savatsy. Dans un autre contexte, ce même groupe vocal s'accompagne en frappant des mains (plage 7, chant exécuté durant une séance de guérison appelée takasy), ou par le jeu du tambour langoro (plage 15, séance de guérison langoro bilo). Le déroulement de ces chants est caractérisé par la répétition de vers courts (plage 1 ; la traduction des paroles n'est pas donnée), ou la transformation graduelle d'un énoncé (plages 7 et 15) assurée par la soliste et reprise par le chœur.

5 Le chant de travail exécuté par deux femmes est magnifique (plage 10, chant de tressage de natte; la transcription des paroles est donnée). Bien que les deux voix soient véritablement différenciées sur les plans mélodique et rythmique, leur parcours semble être intimement lié. L'homogénéité des timbres et la faible intensité des voix, se détachant du bruissement de la paille en arrière plan, accentuent le caractère confidentiel de cette rencontre vocale.

6 Une partie relativement importante du disque (plage 5, 9'03") est consacrée au genre vocal sarandra (" chant de veillée funéraire ») pour deux voix d'hommes. Cette fois-ci, le commentaire du livret renseigne utilement sur le contexte d'exécution de ce répertoire, typiquement antanosy selon l'auteur. Trois textes sont traduits et correspondent visiblement à trois chants différents (les titre donnés sont Eo aminao, « Je te quitte », Récit de la mort d'une personne tuée par les malaso (voleurs de zébus), et Pela aho $e$, « Je suis une femme »). Peut-être aurait-il été nécessaire d'indexer séparément ces trois chants puisqu'il est impossible de les délimiter à l'oreille, tellement le matériau musical est riche et varié. On entend alternativement une polyphonie à deux voix et un soliste. Dans les sections "duo ", les voix sont installées dans un registre relativement aigu, et les deux parties sont homorythmiques sans être strictement parallèles. La voix la plus aiguë développe une ligne mélodique, l'autre étant moins mobile. Ce traitement polyphonique rappelle le bourdon, sans toutefois en avoir les caractéristiques puisque la voix grave ne reste pas à une hauteur fixe. Puis, l'une des voix se tait pendant que l'autre entonne un chant dont l'esthétique contraste fortement avec ce qui précéde; le chanteur fait entendre à lui seul un très riche éventail de techniques vocales. Le contraste des timbres (voix gutturale dans l'aigu, profonde dans le grave), des registres (ligne mélodique descendante entrecoupée de petits cris aigus) et des dynamiques mérite réellement toute l'attention de l'auditeur.

7 Les extraits où sont réunis à la fois voix et instruments de musique polyphoniques sont eux aussi captivants. Sur les plages 8 et 16, une voix d'homme est accompagnée par la 
cithare sur caisse marovany et par le hochet kantsa. Le joueur de cithare est particulièrement virtuose ; il fait preuve d'une telle maîtrise de son instrument, qu'il est capable à lui seul de faire entendre plusieurs plans sonores (polyrythmie et lignes mélodiques raffinées sont mises en valeur par la rapidité du tempo et le contraste des dynamiques). Son jeu occupe une telle place dans le trio, que l'extrait de la plage 3 donne réellement l'impression que c'est la voix qui accompagne le jeu de la cithare, et non pas l'inverse comme le dit Victor Randrianary. La même impression d'accompagnement vocal se dégage des deux pièces pour chœur de femmes et ensemble de "sifflets monotonaux " kololoky (plage 9 et 17). Cet ensemble peut par ailleurs être entendu à la plage 4 . La description organologique qu'en donne Randrianary est obscure, et la photographie à laquelle il se réfère pour la compléter (p. 12) n'éclaire malheureusement pas le lecteur ! Comme pour chacune des seize autres notices, les nom, âge et appartenance ethnique de chaque interprète figurent en tête du texte. On constate ainsi que dix personnes font partie de l'ensemble instrumental (constitué de "sifflets et sifflements»). Sachant que chacun des aérophones n'émet qu'une seule hauteur de son et que les musiciens pratiquent entre eux la technique du hoquet, l'auditeur peut s'interroger sur l'organisation des dix parties entre elles. On aurait souhaité avoir un minimum d'informations sur le système musical lui-même.

On constate encore une fois l'intérêt musical des répertoires instrumentaux et la qualité des musiciens dans les deux duo de flûtes sosoly (à embouchure terminale biseautée, maintenue obliquement) (plages 2 et 14). La rapidité et la précision des attaques sont remarquables pour ce type de flûte; le jeu des musiciens est peut-être facilité par l'utilisation des matériaux de récupération pour la fabrication de ces flûtes (homogénéité et rigidité caractérisent le plastique et le métal) comparativement au bambou traditionnellement employé. La plage 12 fait entendre une intéressante association de timbres: celui de la conque antsiva, sourd et dont le spectre est plutôt riche en harmoniques graves, auquel se superposent des sifflements aigus.

9 Ce panorama de la musique antanosy est complété par deux extraits de musique pour «xylophone sur jambes » atragnatra (l'instrument est uniquement constitué de lames de bois accordées entres elles que l'instrumentiste dépose - selon un ordre interchangeable - perpendiculairement sur ses deux jambes allongées) (plages 6 et 13), et de l'arc musical jejolava (plage 11). L'atragnatra, qualifié par l'auteur d'instrument archaïque, est beaucoup moins répandu que les instruments précédents et est exclusivement joué par deux femmes ou deux jeunes filles dans un contexte de divertissement. La pièce "Milahandra est prête " (plage 13) illustre la composition particulière du répertoire associé à l'atragnatra: sur un tempo rapide, les deux instrumentistes répètent un court motif mélodique dans lequel toutes les notes ont la même valeur rythmique. L'ajout ou le retrait, en cour de jeu, d'une note (ou de quelques-unes) dans ce motif modifie sensiblement notre perception globale de l'événement sonore. Enfin, le chanteur s'accompagnant de l'arc musical jejolava semble ne pas déployer la même énergie que les autres musiciens enregistrés : son jeu est un peu mou, comme si son instrument était fatigué.

Dans ce disque, l'auteur a voulu faire entendre des répertoires musicaux « devenus d'une rareté exceptionnelle, [...] que mêmes les jeunes générations antanosy ignorent aujourd'hui » (p. 4). L'intérêt spécifique de ces documents et leur originalité sont donc évidentes, mais peut-être aurait-il été utile de préciser l'importance socioculturelle de ces 
musiques par rapport aux deux autres répertoires instrumentaux très connus: la musique pour cithare tubulaire valiha ${ }^{1}$ et la musique pour vièle lokanga ${ }^{2}$.

11 Cette publication a le très grand mérite de porter à la connaissance du public l'extrême richesse des répertoires musicaux antanosy, et trouverait sa place dans une éventuelle anthologie de la musique malgache. Cependant, les commentaires de Victor Randrianary manquent parfois de clarté et de rigueur du point de vue de la description organologique, et sont quasiment muets en ce qui concerne l'analyse musicale. Une réflexion sur la musique et sur les nombreux aspects sonores que propose ce disque, qui aurait pu être opportunément éclairée par quelques notations musicales, fait défaut. Néanmoins, on doit reconnaîre que la tâche est immense, et que ces lacunes sont liées au fait qu'il n'existe jusqu'à maintenant presque aucune étude ethnomusicologique approfondie sur la musique des Antanosy, et plus généralement sur celle des peuples du sud de Madagascar.

\section{NOTES}

1. Ce type d'instrument est connu des Antanosy comme le montre, par exemple, les enregistrements publiés par C. Duvelle et M. Razakandraina, Valiha Madagascar, face A plage 7 (OCR 18, 1964 / rééd. en 1982).

2. Instrument qui occupe une place centrale dans les pratiques musicales du sud de Madagascar, et qui est joué par les Antandroy et les Bara, voisin géographiquement des Antanosy. 